\title{
Controlling the assembly of hydrophobized gold nanoparticles at the air-water interface by varying the interfacial tension
}

\author{
Shweta Gupta ${ }^{\text {a }}$, Nahar Singh ${ }^{\text {a }}$, Murali Sastry ${ }^{\mathrm{b}}$, Rita Kakkar ${ }^{\mathrm{c}}$, Renu Pasricha a,* \\ a Material Characterization Division, National Physical Laboratory, New Delhi-110012, India \\ ${ }^{b}$ Tata Chemical Innovation Center, Anmol Pride, Baner Road, Pune-45, India \\ Department of Chemistry, Delhi University, Delhi-110007, India
}

A B S T R A C T

Controlled assembly is the key to harness the nanoscale properties of nanoparticles in most technological applications and it has been an important challenge as it leads to the manipulation of interparticle properties. The present work depicts the control of the assembly of nanoparticles in the monolayers by evaporation kinetics and particle interactions at the air-liquid interface. In the presence of attractive particle-particle and particle-monolayers interactions, nanoparticles self assemble into a superlattice structure upon drying from a colloidal suspension on to the preformed lipid monolayers. This self-assembly mechanism produces monolayers with long-range ordering. However, rapid dewetting and high rate of evaporation can significantly undermine the extent of ordering. Using gold nanoparticles as vehicles for experimentation and by changing the monolayers and solvent, we here demonstrate that the extent of ordering of nanoparticles can be controlled.

\section{Introduction}

The fact that nanoparticles and assemblies exhibit unique electrical, optical, magnetic, and catalytic properties is not only because of the dramatic increase in the surface area/volume ratio as the particle size is reduced but also because of the emergence of collective and nanoscale properties as a result of the interparticle arrangement or assembly. It is the exploration of such nanostructures that has captured the growing interests of research at the interfaces of chemistry, physics, biology, and materials science. An important challenge in harnessing the nanoscale properties of nanoparticles in technological applications involves controlled assembly. The ability to engineer the nanoscale properties is essential and has been the subject of extensive reviews in recent years [1-5]. Directed selfassembly of nanoparticles opens new avenues of technology through controlled fabrication of nanoscopic materials with unique optical, magnetic, and electronic properties [6-8]. The understanding of the directed/mediated assembly allows for fine tuning of the interparticle properties at the molecular level. This level of control also provides an advanced understanding of the phenomena of aggregation, which has long been a subject of research on small-sized particles.

Construction of a macroscopic assembly or superlattice requires availability of stable building blocks of nanoparticles having uniform sizes and shapes and suitable surface capping material that allows interparticle separation. Several approaches have been adopted in order to obtain 2D and 3D self assembled nanostructures [9-24]. The Langmuir-Blodgett (LB) technique is one of the potential ways to assemble the nanoparticles with well-defined structure and thickness controlled at molecular level. Metal, metal oxide and semiconductor nanocrystals have been shown to self assemble into ordered structures upon evaporation of the solvent on a solid substrate or at the water-air interface [25-27]. Experimentally, periodicity with long-range ordering is hard to achieve not only because of the intrinsic particle size distribution but also because of the dewetting of the solvent during the self-assembly process.

Several approaches have been adopted in order to obtain 2D and 3D self assembled nanostructures [9-13,28,29]. However, these nanoparticle assemblies generally lack long-range ordering. In this article we describe how solvents, surfactants and surface pressure play a role in the formation of 2D superlattice of gold nanocrystals. We demonstrate that, by controlling the spreading and subsequent evaporation process of the solvent in the presence of preformed lipid monolayer, long-range-ordered nanocrystal superlattices can be prepared routinely, with domain size of several microns. Two different surfactants, anionic (stearic acid, StA) and cationic (octadecylamine, ODA), have been used to form the monolayer. Presented subsequently are details of our study.

\section{Experimental details}

In a typical experiment, gold nanoparticles were synthesized by sodium borohydride reduction of $10^{-4} \mathrm{M}$ aqueous solution of 
chloroauric acid, that results in a clear ruby red coloured gold nanoparticles solution of $\mathrm{pH} 9$ with a particle size of $9 \pm 1.5 \mathrm{~nm}$ [30]. Vigorous shaking of these aqueous gold nanoparticles with $10^{-3} \mathrm{M}$ chloroform solution of ODA resulted in the rapid transfer of these gold nanoparticles from aqueous into chloroform phase, imparting red colour to the originally colourless chloroform phase. The chloroform phase was separated and was dried to form powder of the hydrophobized gold nanoparticles (referred to as Au-ODA). This powder was washed several times with ethanol to remove uncoordinated ODA molecules.

Thin multilayered films of hydrophobized gold nanoparticles were formed using pure deionized water as the subphase on the LB trough. In a typical experiment, the solution of hydrophobized gold nanoparticles was prepared in chloroform and benzene at a concentration of $1 \mathrm{mg} / \mathrm{ml}$. This solution was then spread slowly onto the water surface in a drop wise fashion and was allowed to stand for at least $20 \mathrm{~min}$ for solvent evaporation prior to compression. The film was then compressed by a barrier with moving speed of $20 \mathrm{~cm}^{2} / \mathrm{min}$. Film of nanoparticles was deposited onto carbon-coated copper grids by lifting the substrates vertically under controlled surface pressures. The $\Pi-\mathrm{A}$ isotherm corresponding to pure Au-ODA on water and the representative TEM image are shown in supplementary information Fig. S1(A-B).

StA and ODA were used for the formation of the monolayer on the water sub phase. $75 \mu \mathrm{l}$ of $(1 \mathrm{mg} / \mathrm{ml})$ solution of ODA/StA in chloroform was spread slowly onto the water surface at $\mathrm{pH}$ 7.1. After spontaneous spreading on the sub phase, the film was allowed to stand for at least $20 \mathrm{~min}$ for solvent evaporation prior to isotherm measurements. Monolayer thus formed was compressed to three different pressures of $5 \mathrm{mN} / \mathrm{m}, 15 \mathrm{mN} / \mathrm{m}$ and $20 \mathrm{mN} / \mathrm{m}$ corresponding to the gas, liquid and solid phases. Upon the surface pressure reaching a target value, $50 \mu \mathrm{l}$ of the $1 \mathrm{mg} / \mathrm{ml} \mathrm{mixture} \mathrm{of} \mathrm{hydrophobized} \mathrm{gold} \mathrm{nanoparticles} \mathrm{in}$ solvent was spread on the monolayer (Fig. 1A (schematic)).

\section{Results and discussion}

The spreading behavior of Au-ODA in solvent on the monolayer protected water surface was studied at room temperature by varying the surface pressure and also by using two different solvents chloroform and benzene. Fig. $1 \mathrm{~B}$ and $\mathrm{C}$ shows the photographs of the domain of Au-ODA nanoparticles in chloroform on the StA and ODA monolayer, respectively, at surface pressure $15 \mathrm{mN} / \mathrm{m}$ on the LB

A

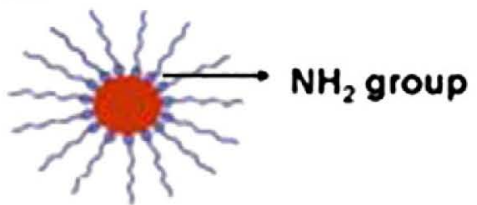

COOH group

\section{hydrophobized Au nanoparticles}

\section{Steric acid molecule hydrophobized Au nanoparticlostadecylamine \\ on subphase \\ molecule}
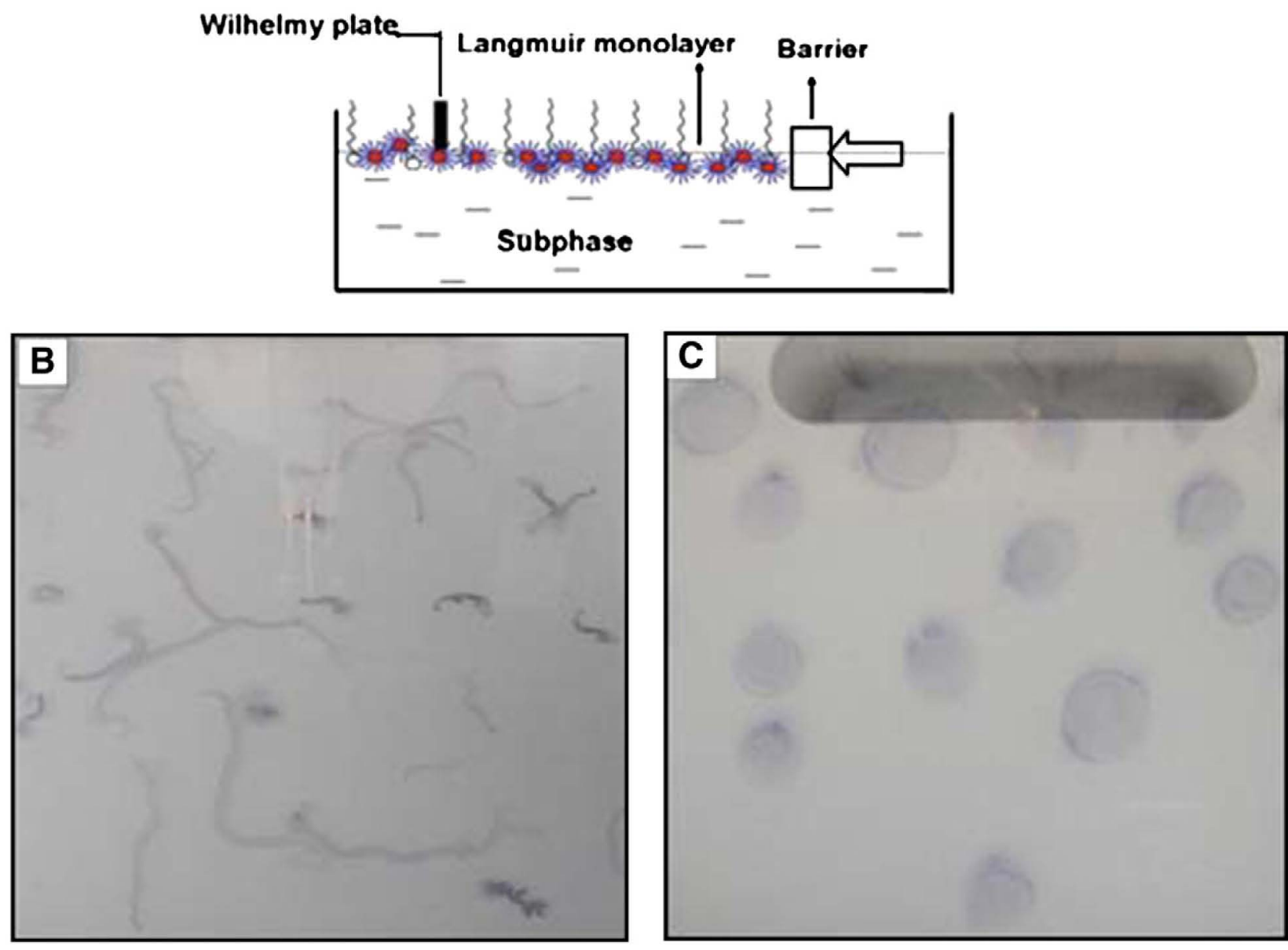

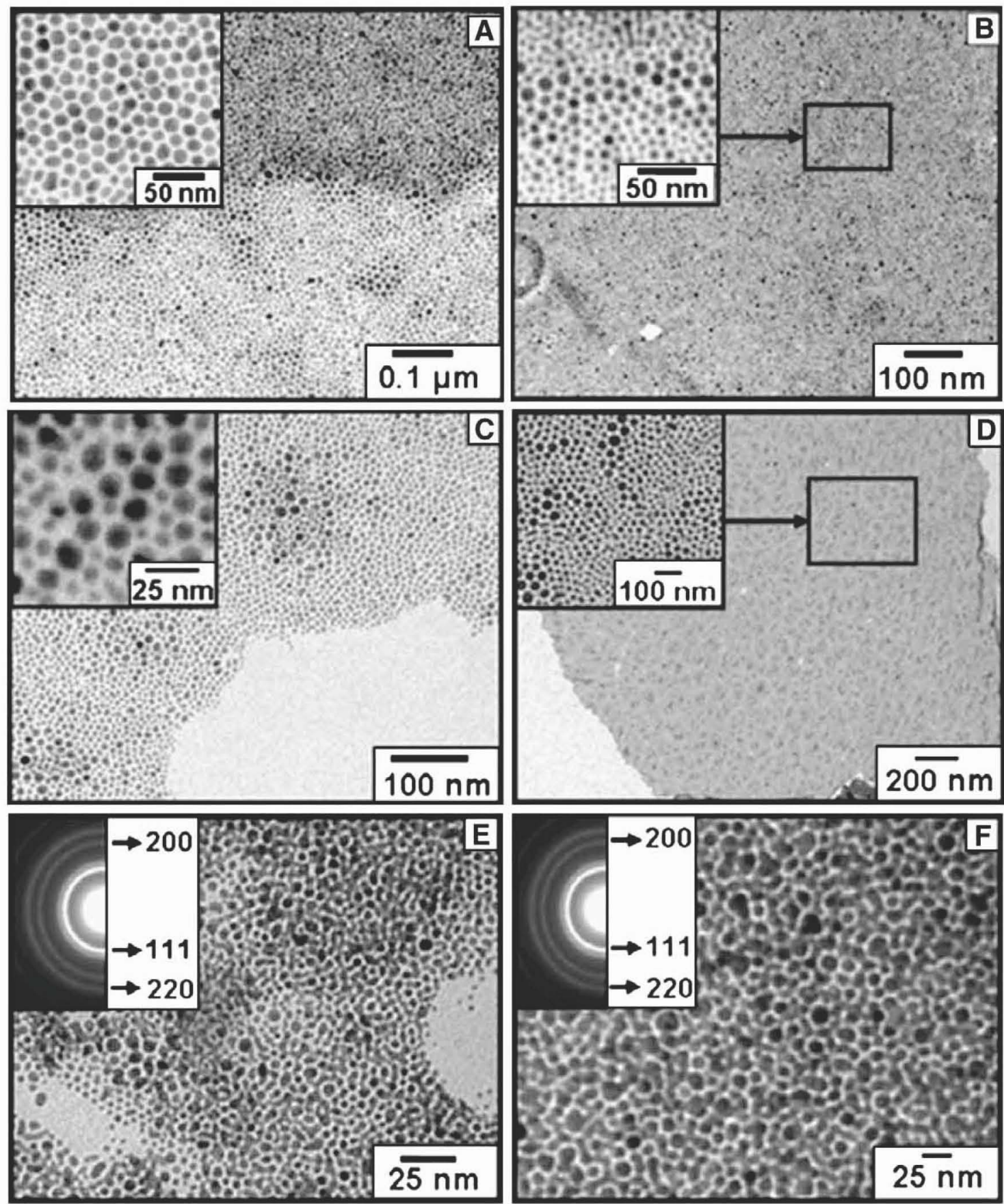

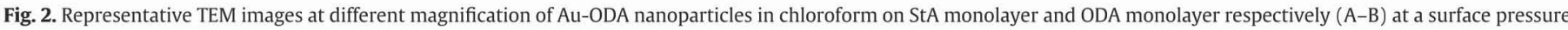

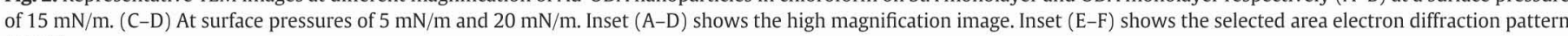
(SAED).

trough. In case of StA monolayer they are tape like, with a clear outer dark line and thin inner areas and in case of ODA monolayer, the domains are circular with an outer line of contact and inner light areas. The structures are different due to difference in wettingdewetting behavior and different interactions of the hydrophobized gold particles with the lipid monolayers.

In the case of Au-ODA in chloroform on StA monolayer, it was observed that the rate of evaporation of the drop (about $5 \mu \mathrm{l}$ ) of AuODA-chloroform solution onto the monolayer decreases with the increase of the surface pressure at which the lipid film is compressed $(5 \mathrm{mN} / \mathrm{m}-20 \mathrm{mN} / \mathrm{m})$ and the spreading of the drop changes from irregular shape resembling the phenomenon of viscous fingering to spherical with formation of multilayers. Only exception being the compression pressure $15 \mathrm{mN} / \mathrm{m}$, where the drop does not spread at all, but contracts in size sitting on the monolayer and then after 2-3 s, it starts to move fast in a jet-like motion onto the surface, leaving a trail of nanoparticle film behind (video recording available as supplementary information). Whereas in the case of Au-ODA in chloroform on ODA monolayer, the spreading and evaporation behavior of the drop at surface pressure of $5 \mathrm{mN} / \mathrm{m}, 10 \mathrm{mN} / \mathrm{m}$ and $20 \mathrm{mN} / \mathrm{m}$ are the same as in the case of StA monolayer but at the surface pressure of $15 \mathrm{mN} / \mathrm{m}$ the drop wets the surface and then evaporates slowly over a time of 3-4 s leaving behind a thin circular domain which resembles the coffee stain pattern. It is well known 

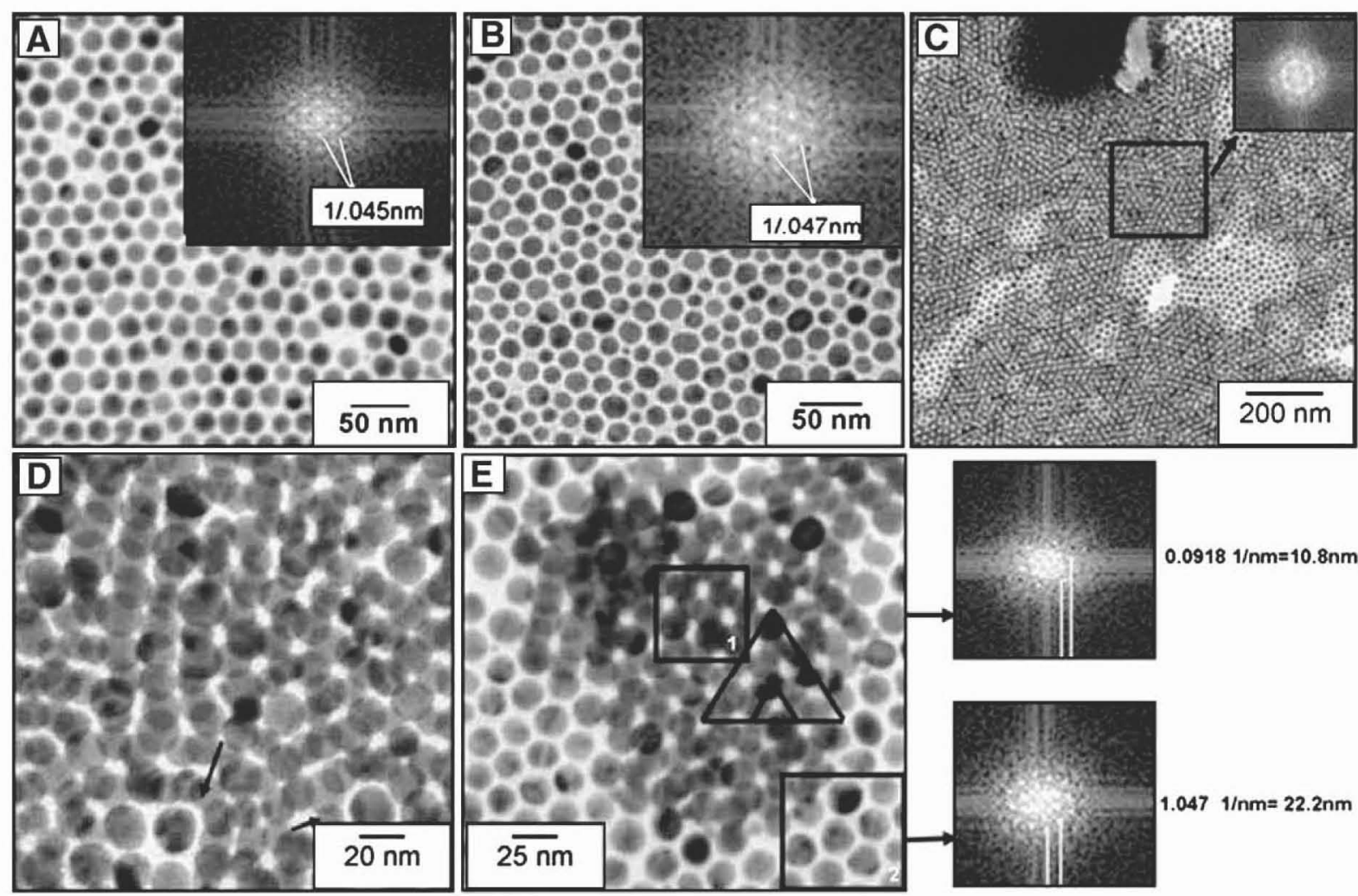

$0.09181 / \mathrm{nm}=10.8 \mathrm{~nm}$

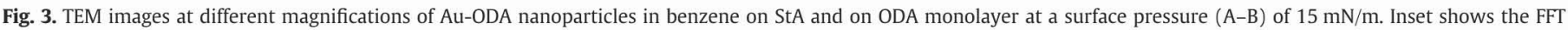

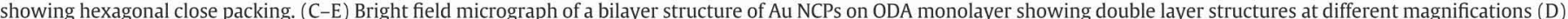

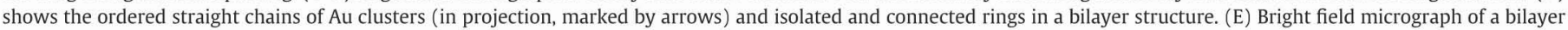
structure of Au NCPs on StA monolayer showing double layer structures. The FFTs from the area marked 1 and 2 of (E) are also shown.

that the evaporation of sessile drops can proceed either through constant contact area and decreasing contact angle or by constant contact angle and decreasing contact area [31]. The case of Au-ODA on the ODA monolayer seems the case of the drop maintaining a constant area.

The BAM study (shown as supplementary information, Fig. S2) conducted to study the transition in the morphology of the StA monolayer with increasing pressure indicated formation of a domain structure similar to the reports for the monolayers of fatty acids [32]. The contrast between the mosaic domains decreases when the surface pressure is increased further. The BAM images of ODA show a different morphology which is continuous. The structure of the lipid monolayer at the interface long with the interaction between the lipid monolayers and the nanoparticles may be responsible for the unusual spreading behavior of the Au-ODA onto the monolayer. In addition to this, chloroform continues to evaporate from the surface at a rapid rate (evaporation rate 11.2 w.r.t. butyl acetate $=1$ ) thus imposing a pressure onto the particles to spread faster in between the gaps of the monolayer. It is worth noting that in a similar case with ODA capped nanoparticles in benzene with all other condition being same, we don't observe a similar phenomenon.

The formation of different assemblies of nanoparticles is supported by direct and visual characterization (TEM measurements). Fig. 2A and $\mathrm{B}$ shows that the TEM images for Au-ODA in chloroform on StA
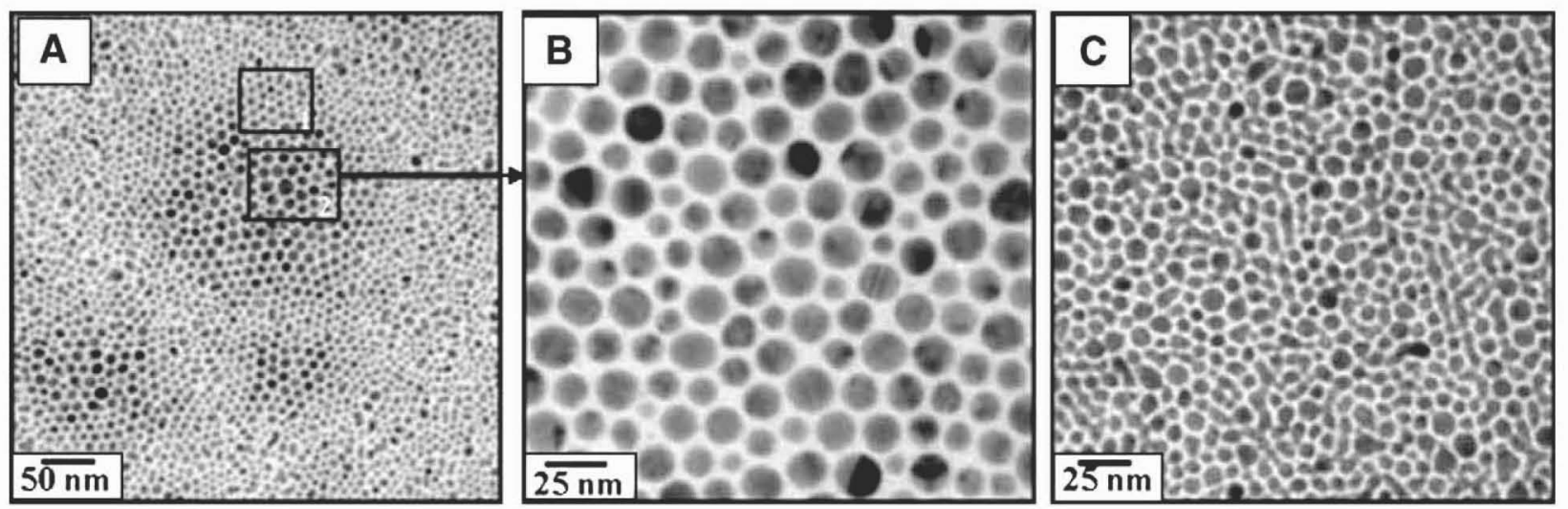

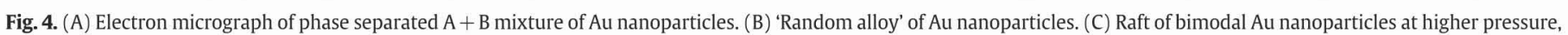
smaller particles have partially sintered and formed a semicontinuous network around the larger particles. 

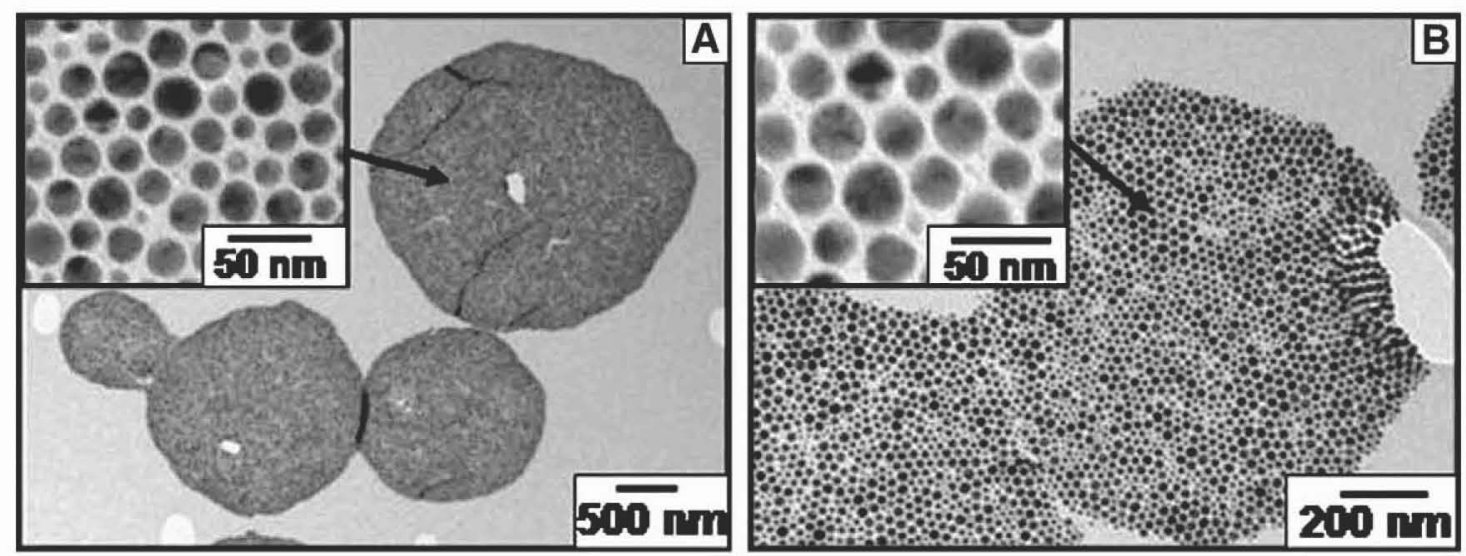

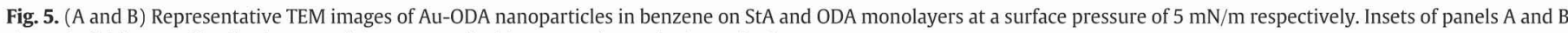
show the high magnification images of the area marked by arrow shown in the main figures.

monolayer and ODA monolayer respectively picked at different surface pressures. From the images it is clearly seen for both the cases (on StA and ODA monolayers) that the monolayer domains are large. The TEM micrographs of gold nanoparticles on StA shows domain having thick outer multilayers (Fig. 2A) whereas on the ODA monolayer they show uniform large domain (Fig. 2B). This may be due to the interaction between the particles in solvent and the lipid monolayer on the subphase. On varying the surface pressure of the preformed lipid monolayer we got completely different arrangement of the nanoparticles and spreading behaviors. Fig. 2C, D and E, F shows the TEM images of Au-ODA in chloroform lipid monolayers, at surface pressure $5 \mathrm{mN} / \mathrm{m}$ and $20 \mathrm{mN} / \mathrm{m}$ respectively. Inset of the figure shows the selected area electron diffraction pattern which could be indexed to fcc structure of gold lattice. There is a transition from incomplete coverage of the surface at low surface pressure to almost complete coverage, eventually leading to fused interlinked superstructures of the monolayer at sufficiently high pressure.

In order to understand the role of rate of evaporation of the solvent, similar experiments were carried out by spreading the Au-ODA nanoparticles in benzene onto the StA and ODA monolayers. Fig. 3A and $B$ shows the TEM images respectively of the ODA capped gold nanoparticles spread onto the StA and ODA monolayers at the surface pressure of $15 \mathrm{mN} / \mathrm{m}$. The interaction of Au-ODA nanoparticles with the StA and ODA present in the monolayer is different and it is reflective in the range of ordering displayed by the fast Fourier transform (FFT), inset of Fig. $3 \mathrm{~A}$ and $\mathrm{B}$ which shows an ordering up to the third order and the 6fold symmetry of the structure, respectively in the presence of StA and ODA monolayers. This also indicates the hexagonal close-packed lattice [33] arrangement with interparticle spacing being 21-22 , which agrees with the single molecular length of ODA.

At higher pressures, a transition from monolayer coverage of the surface to multiple layers of highly ordered nanoparticles is observed unlikely. Fig. 3C-E shows the TEM images of Au-ODA in benzene on ODA and StA monolayers at a surface pressure of $20 \mathrm{mN} / \mathrm{m}$. The structure of these multilayers are different from the structures reported earlier $[10,34,35]$, where the second-layer nanocrystals sit on the two-fold saddle sites between the first-layer particles, forming either line or ring structure. However, in our highly ordered bilayers, the second-layer particles position themselves almost exclusively on the three-fold sites of the monolayer underneath much like hard sphere packing. Three-fold sites are more favourable and stable and hence we are able to achieve a perfect formation of well-ordered bilayers on increasing the surface pressure.

It is well known that electron diffraction is dominated by dynamic scattering for specimens thicker than $2 \pm 3 \mathrm{~nm}$ [36]. In nanocrystal superlattice (NCS) analysis, dynamic scattering is also inevitable. Fig. 3C-E shows bright-field TEM images of an Au NCS. The particle size is seen at the places in the specimen where the number of stacked layers is likely to be one or two. However, in the region with multilayer stacking, fringes considerably smaller than the interparticle distance are observed. A Fourier transform (FFT) of this image is given in the inset of Fig. $3 \mathrm{C}$ and also for regions 1 and 2 of Fig. 3E. The FFT is shown as the inset of Fig. $3 \mathrm{C}$ and also for regions 1 and 2 of Fig. $3 \mathrm{E}$. The FFT of region 2 reveals the presence of the second-order diffraction spots from the NCS. The interference of these beams with the central transmitted beam or the beams located at the opposite side in the pattern could give rise to fringes with spacing less than the actual distance between the particles. It is apparent by the distance measured using Digital Micrograph where the single layer gives the particle to particle distance to be $22.2 \mathrm{~nm}$ (Fig. 3E, region 2), and $10.8 \mathrm{~nm}$ from the region 1 (Fig. 3E) showing double layer.

In both cases of solution of gold nanoparticles, it is noticed that besides the range of ordering in the monolayers, the bimodal distributed gold particles also show some tendency to form ordered structures by a process involving spontaneous size segregation $[37,38]$. It is clearly seen that gold nanocrystals of different, well-defined sizes organize themselves spontaneously into complex, ordered twodimensional array. We observe three types of organization: first, size-segregated regions, each containing hexagonal-close-packed monodisperse particles (Fig. 4A) showing an organization of an approximately bimodal ensemble of particles for which $\mathrm{R}_{B} / \mathrm{R}_{A} \approx 0.47$, where $R_{A}$ and $R_{B}$ are the radii of the nanoparticles [39]; second, $a$ structure in which particles of two different sizes occupy random positions in a pseudohexagonal lattice (Fig. $4 B$ ) that is if $R_{B}$ and $R_{A}$ are very similar, the structure formed approximates to an intimate random alloy [40]; and third, (Fig. 4C) a structure in which smaller particles have partially sintered, and formed a semi-continuous network around the larger particles. As is in the case of chloroform, the role of surface pressure in the assembly of nanoparticles with benzene being the solvent of dispersion was also studied. Fig. $5 \mathrm{~A}$ and $\mathrm{B}$ shows the TEM images of Au-ODA in benzene spread onto STA and ODA monolayers at $5 \mathrm{mN} / \mathrm{m}$ surface pressure. It is clear that for benzene as solvent, the surface pressure is also an important factor in controlling the extent of monolayer formation but not for the longrange ordering of particles. It can be clearly seen that at the surface pressure $5 \mathrm{mN} / \mathrm{m}$ close-packed super lattices are formed without the appearance of particle aggregation. However at higher pressures, a transition from monolayer coverage of the surface to multiple layers of highly ordered nanoparticles is observed. The third kind of arrangement is seen in the case of increased pressure condition. This phenomenon can be attributed to the fact that the smaller particles have fused together as it may be energetically favourable for the smaller Au particles to minimize their surface energy $[31,41,42]$ by sintering under higher barrier pressure. The micrograph shows an ordered bimodal raft wherein the $13 \pm 1 \mathrm{~nm}$ diameter particles are still intact, whereas the $9 \pm 1 \mathrm{~nm}$ particles have spread. The smaller 
particles have effectively connected to form a semi-continuous network in which the regular isolated ODA capped larger particles are embedded. A further contributing factor is the strong dependence of sintering rate on particle radius. All other conditions (such as temperature, ambient humidity) being equal, the smaller particles are expected to sinter at a relative rate of approximately $\left(R_{A} / R_{B}\right)$. This raises the possibility that the size-dependence of particle stability might be exploited for nanopatterning; the observations described here indicate the possibility of manipulating the composition and structure of nanoscale materials by simply adjusting the size ratios and relative proportions of their constituents.

As described earlier, the as-synthesized nanoparticles are dispersed in liquid, in which size selection according to mass is happening. NCSs are formed by depositing droplets of passivated nanocrystals on the aqueous subphase having a predeposited charged lipid monolayer. The diffusion of the nanocrystals on the subphase is possibly driven by hydraulic forces as drying takes place. Thus, wetting problem, viscosity, and limiting concentration of the nanocrystals determine the types of arrangement of nanoparticles.

\section{Summary}

We conclude that in the absence of the monolayer on the water sub phase, the liquid drop spreads fast into a very thin film and evaporates before the long-range ordering or super lattice formation. Only when the interactions between particles are strong enough and they get time to assemble, a perfect monolayer is formed. In analogy to molecular crystallization, it is reasonable to propose that better super lattices, larger and with fewer defects, are obtained when they form slowly. We have shown that keeping all other parameters constant and varying the pressure can change the evaporation rate and lead to the formation of long-range ordering and subsequently to super lattices. The interactions between ODA capped Au nanoparticles and lipid molecules present in the monolayers are responsible for the formation of thermodynamically stable well-ordered assembly at the air-water interface by balancing of steric repulsion interaction and strong hydrophobic interactions between the hydrophobic part of the lipid molecules present in the monolayer and on gold nanoparticles.

Supplementary materials related to this article can be found online at doi:10.1016/j.tsf.2010.08.046.

\section{Acknowledgements}

Renu Pasricha thanks Director NCL, where the work was initiated and Director NPL for the permission to carry out the work. Shweta thanks the Department of Science and Technology (DST), Govt. of India for a research fellowship.

\section{References}

[1] A.P. Alivisatos, Science 271 (1996) 933.

[2] R.W. Murray, Chem. Rev. 108 (2008) 2688.

[3] N.L. Rosi, C.A. Mirkin, Chem. Rev. 105 (2005) 1547.

[4] S.K. Ghosh, T. Pal, Chem. Rev. 107 (2007) 4797.

[5] M.C. Daniel, D. Astruc, Chem. Rev. 104 (2004) 293.

[6] L. Brus, Appl. Phys. A 53 (1991) 465.

[7] A.P. Alivisatos, Adv. Mater. 10 (1998) 1297.

[8] S.A. Empedocles, R. Neuhauser, M.G. Bawendi, Nature 399 (1999) 126.

[9] N. Kimizuka, T. Kunitake, AdV. Mater. 8 (1996) 89.

[10] Z.L. Wang, Adv. Mater. 10 (1998) 13.

[11] M. Sastry, Handbook of surfaces and interfaces of materials, in: H. Nalwa (Ed.), Volume 3: Nanostructured Materials, Micelles and Colloids, Academic Press, San Diego, 2001, p. 87, Chapter 2.

[12] M.-P. Pileni, J. Phys. Chem. B 105 (2001) 3358.

[13] S. Sun, C.B. Murray, D. Weller, L. Folks, A. Moser, Science 287 (2000) 1989.

[14] T. Nakanishi, B. Ohtani, K. Uosaki, J. Phys. Chem. B 102 (1998) 1571.

[15] E.W.L. Chan, L. Yu, Langmuir 18 (2002) 311.

[16] E. Hao, T. Lian, Chem. Mater. 12 (2000) 3392.

[17] S. Malynych, I. Luzinov, G. Chumanov, J. Phys. Chem. B 106 (2002) 1280.

[18] S. Sun, S. Anders, H.F. Hamann, J.U. Thiele, J.E.E. Baglin, T. Thomson, E.E. Fullerton, C.B. Murray, B.D. Terris, J. Am. Chem. Soc 124 (2002) 2884.

[19] M. Trau, D.A. Saville, I.A. Aksay, Science 272 (1996) 706.

[20] H.X. Li, M.Z. Lin, J.G. Hou, J. Cryst. Growth 212 (2000) 222.

[21] T. Teranishi, M. Hosoe, T. Tanaka, M. Miyake, J. Phys. Chem. B 103 (1999) 3818

[22] N. Chandrasekharan, P.V. Kamat, Nano Lett. 1 (2001) 67.

[23] M. Sastry, M. Rao, K.N. Ganesh Acc, Chem. Res. 35 (2002) 847.

[24] M. Sastry, in: F. Caruso (Ed.), Colloids and Colloid Assemblies, Wiley-VCH, Weinheim, 2004, p. 369, Chapter 12.

[25] M. Sastry, V. Patil, K.S. Mayya, D.V. Paranjape, P. Singh, Sainkar, Thin Solid Films 324 (1998) 239.

[26] A. Swam, A. Kumar, P.R. Selvakannan, S. Mandal, M. Sastry, J. Colloid Int. Sci. 260 (2003) 367.

[27] H. Weller, Angew. Chem., Int. Ed. Engl. 35 (1996) 1079.

[28] P. Cheng, D. Li, Z. Yuan, Y. Zou, D. Yang, Materials chemistry and physics 111 (2008) 271.

[29] Y.K. Park, S.H. Yoo, S. Park, Langmuir 23 (2007) 10505.

[30] V. Patil, R.B. Mlvankar, M. Sastry, Langmuir 15 (1999) 8197.

[31] R.D. Deegan, O. Bakajin, T.F. Dupont, G. Huber, S.R. Nagel, T.A. Witten, Nature 389 (1997) 827.

[32] G.A. Overbeck, D. Mobius, J. Phys. Chem. 97 (1993) 7999.

[33] M. Giersig, P. Mulvaney, Langmuir 9 (1993) 3408.

[34] B.A. Korgel, S. Fullam, S. Connolly, D. Fitzmaurice, J. Phys. Chem. 102 (1998) 8379.

[35] J. Fink, C.J. Kiely, D. Bethell, D.J. Schiffrin, Chem. Mater. 10 (1998) 922.

[36] Z.L. Wang, Elastic and Inelastic Scattering in Electron Diffraction and Imaging, Plenum, New York, 1995.

[37] P.C. Ohara, D.V. Leff, J.R. Heath, Gelbart, Phys. Rev. Lett. 75 (1995) 3466.

[38] M.J. Murthy, Z.L. Wang, R.L. Whetten, Phil. Mag. Lett. 75 (1997) 321.

[39] M.J. Murray, J.V. Sanders, Phil. Mag. A 42 (1980) 721.

[40] W. Hume-Rothery, R.E. Smallman, C.W. Haworth, The Structure of Metal and Alloys, Metals and Metallurgy Trust, London, 1969.

[41] L. Motte, F. Billoudet, M.P. Pileni, J. Phys. Chem. B 99 (1995) 16425.

[42] C.J. Kiely, J. Fink, M. Brust, D. Bethell, D.J. Schiffrin, Nature 396 (1998) 444. 